\title{
Portal venular constriction during anaphylactic shock in anesthetized rats
}

\author{
Masataka Kitadate $^{1 \#}$, Toshishige Shibamoto ${ }^{2 \#}$, Mikihiro Tsutsumi ${ }^{3}$, and Hisao Tonami ${ }^{1}$ \\ Departments of ${ }^{1}$ Radiology, ${ }^{2}$ Physiology II, and ${ }^{3}$ Gastroenterology, Kanazawa Medical University, Uchinada 920-0293, Japan \\ (Received 12 November 2012; and accepted 25 December 2012)
}

\begin{abstract}
Hepatic venoconstriction occurs in rat anaphylactic hypotension. However, the exact venoconstrictive site remains unknown, and we therefore attempted to determine its location by using hepatic venography and histology. Anaphylaxis was induced in anesthetized Sprague-Dawley rats by i.v. administration of ovalbumin antigen. Venography of the portal vein $(n=8)$ was obtained at baseline and maximal hepatic venoconstriction. We separately examined photomicrographs of the liver sections. Along with systemic hypotension, portal venous pressure increased to a peak of $28 \pm 3 \mathrm{~cm}$ $\mathrm{H}_{2} \mathrm{O}$ at 2 min after antigen injection. Post-antigen portal venography revealed that $40 \%$ of portal venules (76 vessels/total 188 vessels) with diameters from 160 to $300 \mu \mathrm{m}$ were not visualized, suggesting that stenosis or obliteration occurred distally. The corresponding upstream portal vessels exhibited markedly bulging. Stenosis was also observed in some portal branches with diameters from 180 to $420 \mu \mathrm{m}(9 \% ; 17$ vessels/total 188 vessels). Light microscopically, most portal venules with an estimated baseline diameter less than $110 \mu \mathrm{m}$ showed stenosis, but statistically significant stenosis was found in those with baseline diameters of 20-70 $\mu \mathrm{m}$. In conclusion, anaphylactic hepatic venoconstriction occurs over a wide range of portal veins with diameters less than $420 \mu \mathrm{m}$, and occurs markedly in portal venules with diameters less than $70 \mu \mathrm{m}$ in anesthetized rats.
\end{abstract}

Anaphylactic hypotension is in part caused by a decrease in effective circulating blood volume, which could be caused by vasodilation in conjunction with the peripheral blood pooling, as well as by a shift of intravascular fluid to the extravascular space due to increased vascular permeability (2). In canine anaphylactic shock, hepatic venoconstriction, especially selective constriction of the postsinusoidal hepatic veins, induces pooling of blood in the upstream splanchnic organs, as well as in the liver itself $(5,6$, 20). Likewise, in rat anaphylactic hypotension, hepatic venoconstriction occurs, although the liver blood volume decreases rather than increasing (8, $16,19)$. This anaphylactic hepatic venoconstriction

Address correspondence to: Dr. Toshishige Shibamoto, Professor and Chairman of Physiology II

Department of Physiology II, Kanazawa Medical University, Uchinada 920-0293, Japan

Tel: +81-76-286-2211, Fax: +81-76-286-8010

E-mail: shibamo@kanazawa-med.ac.jp plays a significant role in the genesis of blood pressure fall, based on the finding that the elimination of acute portal hypertension by means of surgical perturbations or portacaval shunting attenuates the antigen-induced systemic hypotension in anesthetized rats $(10,16)$. However, detailed information on the anaphylactic hepatic venoconstriction which causes acute portal hypertension is limited.

The anatomical site for anaphylactic venoconstriction of the liver remains unknown. In a previous work using the hepatic vascular occlusion method, we reported that the portal veins are the primary site of anaphylactic hepatic venoconstriction in isolated perfused rat livers (16). Recently this notion was reinforced by measuring the hepatic venular pressure with a servo-null micropipette pressure measurement technique: anaphylactic venoconstriction in the liver venous circulation occurred prior to the hepatic ve-

\footnotetext{
${ }^{\#}$ These authors contributed equally to this work.
} 
nules, that is, the portal veins or the sinusoids (18). However, the exact constrictive site in the portal veins is not known. Thus the purpose of the present study was to determine the primary site of anaphylactic venoconstriction in the liver by using portal venography. We also morphologically examined tissue sections of the liver to compensate for the shortcomings of venography: namely, if constriction of the small portal veins, i.e., portal venules, is strong, the contrast medium in venography will not reach the venoconstrictive site, resulting in a failure to visualize the venoconstrictive veins (11). In addition, the limit of the spatial resolution in the angiography system was $150 \mu \mathrm{m}$.

\section{MATERIALS AND METHODS}

Animals. The experiments in the present study were approved by the Animal Research Committee of Kanazawa Medical University, and conducted in keeping with the Principles of Laboratory Animal Care (NIH publication No. 85-23, Revised 1996). Eighteen male Sprague-Dawley rats (Japan SLC, Shizuoka, Japan) weighing $542 \pm 41 \mathrm{~g}$ were used in this study. Rats were maintained at $23^{\circ} \mathrm{C}$ under pathogen-free conditions on a 12 : 12-hour dark/light cycle, and allowed food and water ad libitum.

Sensitization. Rats were sensitized by the subcutaneous injection of an emulsion made by mixing equal volumes of complete Freud's adjuvant $(0.5 \mathrm{~mL})$ with $1 \mathrm{mg}$ ovalbumin (grade V; Sigma) dissolved in physiologic saline $(0.5 \mathrm{~mL})$ (16). Non-sensitized rats were injected with complete Freund's adjuvant and ovalbumin-free saline.

Surgical preparation of animals. After two weeks, the rats $(n=8)$ were anesthetized with pentobarbital sodium (50 mg/kg, i.p.), and placed on an angiographic flat-panel detector system (Infinix Celeve-I; Toshiba, Tokyo, Japan). The adequacy of anesthesia was monitored by the stability of blood pressure and respiration under control conditions and during a tail-pinch stimulus. Supplemental doses of anesthetic (10\% of initial dose) were given intraperitoneally if necessary. Systemic arterial pressure (Psa) was recorded from the right carotid artery. The right femoral vein was catheterized for an intravenous injection of antigen. After midline incision, the catheter (ID $0.47 \mathrm{~mm}$, OD $0.67 \mathrm{~mm}$ ) was inserted directly into the main portal vein and the catheter tip was positioned at approximately $1 \mathrm{~cm}$ away from the hepatic hilus. This catheter was connected to a custom-made
Y-type miniature plastic tube (24); one twig end was connected via a water-filled polyethylene tube to the pressure transducer for measurement of the portal venous pressure (Ppv), and the other was used for the intravenous injection of contrast medium. After closure of the abdomen, the baseline measurements were started. The vascular pressures were measured using transducers (TP-400T; Nihon-Kohden, Tokyo, Japan) with the reference level at the right atrium, and recorded with a thermal physiograph (RJC-4124; Nihon-Kohden).

Hepatic venography procedure. For portal venography, warmed nonionic contrast medium (Iomeron 350; Eisai, Tokyo, Japan) was injected into the portal vein with an automated power injector (Medrad Mark V Pro Vis., Warrendale, PA) at $3 \mathrm{~mL} / \mathrm{s}$ with a total injection volume of $1.7 \mathrm{~mL}$, and images were obtained with pulsed fluoroscopy at 14 frames per second. This injection maneuver does not affect the basal hemodynamic levels, although some rats show a transient fall in Psa, followed by a recovery within 2 min.

Baseline venography was performed when hemodynamic parameters were stable for at least $20 \mathrm{~min}$ after surgery. After the baseline measurements, ovalbumin antigen $(0.6 \mathrm{mg})$ was intravenously injected. When the Ppv reached the peak levels, the post-antigen venography was performed.

Measurements of vessel lumen diameter. The images of portal venography were analyzed in the left lateral lobe and the right portion of the middle lobe because the portal branches of these two lobes were depicted without overlap by those of the other lobes, and therefore were suitable for measuring the vessel diameter (13). A sequence of frames was chosen in which the branches of the portal veins were most intensely filled with contrast agent. A measure with a diameter of $100 \mu \mathrm{m}$ was present in the side of each frame and was used for software calibration. The images were magnified and printed on standard $\mathrm{X}$-ray films. The vessel diameter was measured by using a digital micrometer caliper, and, finally, corrected for geometric magnification. The measurement was repeated three times by one examiner on separate days, and the mean values were adopted. The intraclass correlation coefficient for the measured values, as assessed with statistical software (SPSs; IBM Corporation, Endicott, NY), was 0.98.

Histological investigation. In separate experiments, the sensitized $(n=5)$ and non-sensitized control $(n=$ 
5) rats were instrumented for measurement of Ppv and Psa in the same way as in the venography study. After an injection of the antigen, the liver of the sensitized rats was excised when Ppv reached the peak level, while that of the non-sensitized rats was excised at baseline without administration of the antigen for estimation of the basal vessel caliber, as described below. Then the excised livers were immersed into a $10 \%$ buffered formalin solution. The liver specimens were embedded in paraffin, sectioned at a thickness of $4 \mu \mathrm{m}$ and stained with hematoxylin and eosin.

Estimation of the basal vessel caliber of the constricted portal veins of rats suffering from anaphylaxis. According to the methods of Kaneda et al. (11), we estimated the vessel preconstricted caliber of the constricted portal veins in the livers from rats subjected to anaphylaxis. This method is based on the assumption that vessels of a similar diameter have a similar nuclear density, i.e., a similar number of endothelial cell nuclei per unit area or unit length. Under this assumption, the preconstricted size of the constricted venule was estimated by referring to the calibration relation between the endothelial nuclear number and vessel diameter created from the nonsensitized control livers. This was accomplished by counting the endothelial cell nuclei $(\mathrm{N}$, count range from 8 to 72) of the transversely sectioned portal venules selected from the control livers, measuring the inner diameter of each vessel $(\mathrm{D}$, measurement range from 30 to $189 \mu \mathrm{m}$ ), and constructing a nuclear number-diameter (N-D) relation. The number of endothelial cell nuclei was counted under high magnification $(\times 1,000)$ so that the nuclei of the endothelial cells could be precisely differentiated from those of other cells such as neutrophils. When the shape of the vascular lumen was somewhat ellipti$\mathrm{cal}$, the length of a minor axis was adopted as the inner diameter. Using this N-D relation, the pre-antigen vessel diameter of the constricted or dilated portal vessels from livers of the sensitized rats was estimated.

Statistics. All results are expressed as the means \pm SD. For statistical analysis, comparison of the values before and after antigen injection was made by Student's $t$-test. Differences were considered statistically significant at $P<0.05$.

\section{RESULTS}

The venography study

When the antigen was injected intravenously into rats of the portal venography group, Psa decreased significantly from $115 \pm 7$ to $71 \pm 9 \mathrm{mmHg}$, and $\mathrm{Ppv}$ increased from the baseline of $9 \pm 2 \mathrm{cmH}_{2} \mathrm{O}$ to the peak levels of $26 \pm 3 \mathrm{cmH}_{2} \mathrm{O}$ at $119 \pm 12 \mathrm{~s}$ after antigen administration. This finding of acute portal hypertension in anesthetized rats subjected to anaphylactic hypotension is consistent with that of the previous reports (16).

The baseline portal venography showed the tapering of the portal veins as branching off (Fig. 1A). The summarized diameters of each portal branch at baseline are shown in Fig. 2E. The diameter of the 1st branch was $990 \pm 190 \mu \mathrm{m}$, and the diameter decreased to $230 \pm 50 \mu \mathrm{m}$ at the 7 th branch. No significant differences in the portal vein diameter were observed between the 5th and 7th branches, and the peripheral branches distal from the 7th branch could not be identified because the limit of the spatial resolution in the present angiography system was $150 \mu \mathrm{m}$. In contrast, the post-antigen venography, which was performed when Ppv reached the peak level, revealed that the proximal portal branches were enlarged and that the distal branches could not be visualized or showed stenosis at some points. Figs. $1 \mathrm{~A}$ and $1 \mathrm{~B}$ show representative images before and after antigen injection, respectively. In some portal branches, the baseline diameter of $1,010 \mu \mathrm{m}$, as indicated by the white arrowheads (Fig. 1C), increased to $1,070 \mu \mathrm{m}$ after antigen injection (Fig. 1D). In contrast, the other portal branches (black arrowheads) showed stenosis from $240 \mu \mathrm{m}$ at baseline to $170 \mu \mathrm{m}$ after antigen injection. However, many branches that were clearly depicted at baseline were not visible after antigen injection, as indicated by the arrows in Figs. 1C and 1D.

Fig. 2 shows the numbers of portal branches for which the venograms indicated no change (2A), stenosis (2B), and disappearance (vanishment; 2C) after antigen injection as a function of their branch generations from the main portal vein (designated as the 1st branch). The most prominent finding was branch disappearance which accounted for 76 of the total 188 (40\%) vessels analyzed: in these cases, the injected contrast medium was not observed at these levels of the portal branches during anaphylactic venoconstriction. In fact, the vanishment was observed from the 3rd to 7th portal branches, and the mean baseline diameters of these branches was 230 $\pm 40 \mu \mathrm{m}$ (range: from 160 to $300 \mu \mathrm{m}$ ). This vanish- 

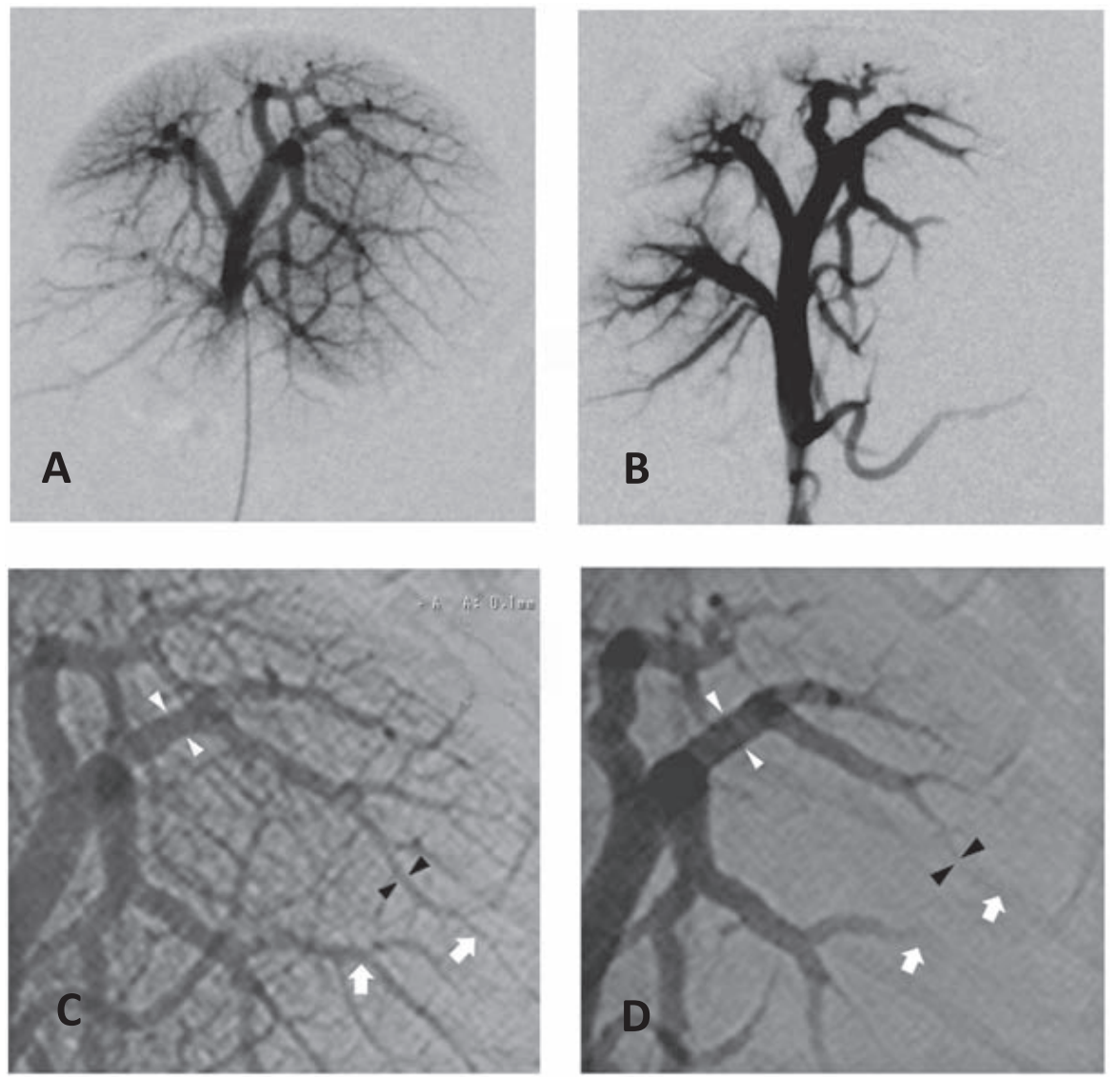

Fig. 1 Portal venogram at baseline $(A)$ and after an injection of the antigen (B). Higher magnification of the left lower lobe at baseline $(C)$ and after antigen injection (D). The white and black arrowheads in panel $D$ show dilatation and stenosis, respectively, as compared with those in panel C. The portal vein indicated by arrows in panel C was not visualized in panel D.

ment during anaphylaxis suggests the presence of marked stenosis or occlusion in the vessels distal to these levels. The corresponding visualized portal venules just upstream from the disappeared ones exhibited bulges whose diameters were greater than the baseline values. As shown in Fig. 2D, enlargement of vessel diameters was observed in the generations from the 1st to 5th (the numbers of portal veins $=92,48 \%$ of total branches), with the corresponding basal diameter of $640 \pm 250 \mu \mathrm{m}$ (from 190 to $1,240 \mu \mathrm{m}$ ). Actually their diameters increased to $790 \pm 300 \mu \mathrm{m}$ after antigen injection. However, apparent stenosis was observed in some portal branches ( $n=17,9 \%$ of total branches examined) from the 3rd to 6th generations, as compared with the corresponding baseline (Fig. 2B). The basal diameter of the portal venules that showed stenosis ranged from 180 to $420 \mu \mathrm{m}$, with a means of $290 \pm 70 \mu \mathrm{m}$. The diameters of these portal veins decreased by $25 \%$ to $220 \pm 50 \mu \mathrm{m}$ at maximal hepatic venoconstriction.

\section{Histological examination}

The liver tissue samples of the sensitized rats subjected to anaphylaxis were obtained at the time of maximal hepatic venoconstriction, i.e., at $120 \pm 12 \mathrm{~s}$ after antigen injection. The Ppv rose to a peak of 30 $\pm 4 \mathrm{cmH}_{2} \mathrm{O}$ from the baseline value of $9 \pm 2 \mathrm{cmH}_{2} \mathrm{O}$, and the Psa decreased to $67 \pm 9 \mathrm{mmHg}$ from the baseline value of $110 \pm 10 \mathrm{mmHg}$. The samples of the non-sensitized control rats were obtained at baseline without antigen injection. Fig. 3 shows representative examples of the photomicrographs of the liver sections from the non-sensitized control and sensitized rats. In liver tissues obtained from nonsensitized rats (Figs. 3A, 3B), as the number of endothelial nuclei increased, the vessel diameters increased: the diameter of the portal veins with nuclear numbers of 23 and 42 selected from the control livers in Figs. 3A and 3B were 51 and $94 \mu \mathrm{m}$, respectively. Fig. 4 shows the N-D relation obtained by using the control rat data collectively. The collective data yielded a linear relationship of $\mathrm{D}=2.27 \times \mathrm{N}$ 

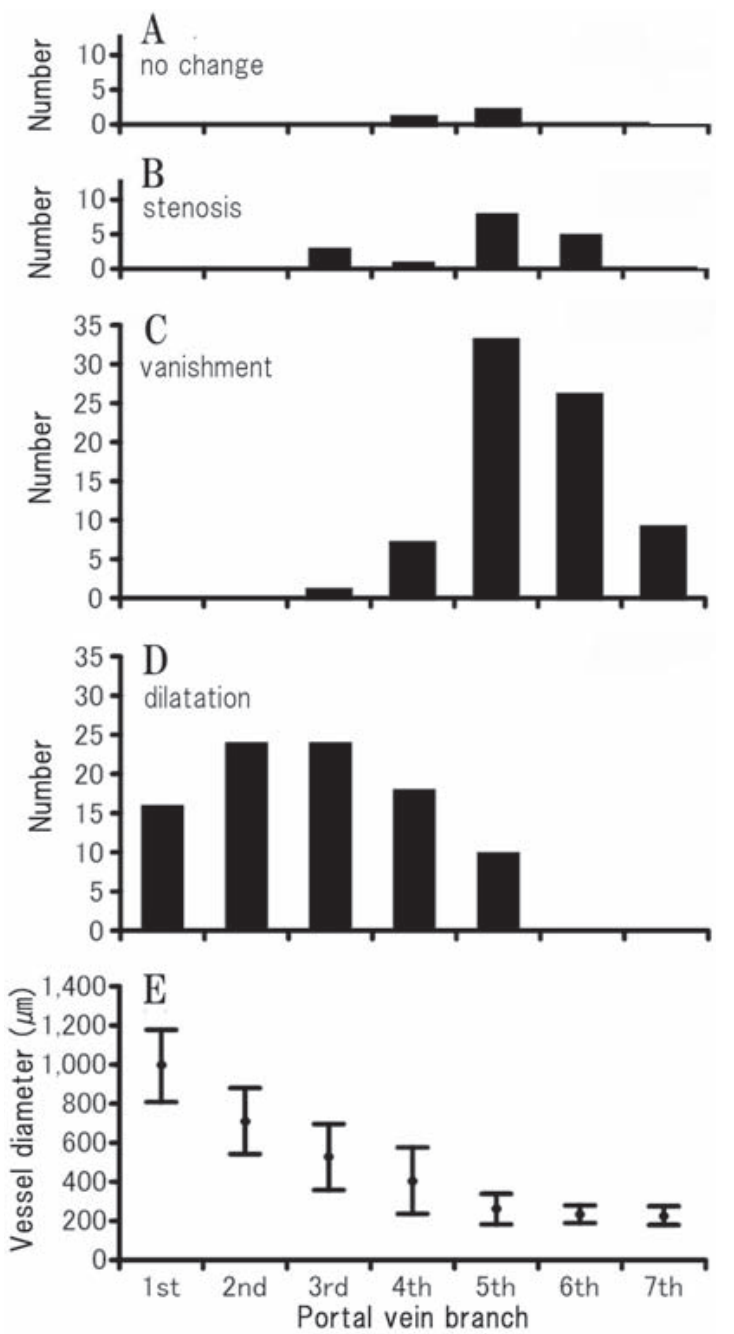

Fig. 2 The numbers of portal vessels that showed no change (A), stenosis (B), vanishment (C), and dilatation (D) as a function of the portal branches at maximal portal venoconstriction after an injection of the antigen. We analyzed a total of 188 portal vessels. The lowest panel (E) shows the diameters of the portal vessels at baseline. The results are shown as the means $\pm \mathrm{SD}$.

(Fig. 4) with a correlation coefficient $r$ of 0.973 . In contrast, in sections of the anaphylactic liver, the portal venules exhibited constriction: the diameters of the constricted portal veins in Figs. 3C and 3D, which exhibited 23 and 42 nuclei of endothelial cells, were 26 and $60 \mu \mathrm{m}$, respectively. These lumen diameters were used to calibrate the preconstricted diameters, giving values of 65 and $95 \mu \mathrm{m}$, respectively. In addition, the constricted portal venules were densely lined with endothelial cells and closely and heavily surrounded by perivascular smooth muscle cells, forming a seemingly "thickened" media with the vascular lumen considerably reduced
(Fig. 3C). Fig. 5A shows the plots of the vessel diameter in the anaphylaxis group as a function of the predicted baseline diameter, where the vessel diameter after antigen injection was expressed as a percentage of the baseline diameter predicted by the $\mathrm{N}-\mathrm{D}$ relation (Fig. 4). Fig. 5B shows the summarized data of the portal vein diameter after antigen injection. The diameter of portal venules with predicted baseline diameters of 20-30, 30-40, 40-50, 50-60 and $60-70 \mu \mathrm{m}$ significantly decreased to 53 $\pm 11 \%, 65 \pm 10 \%, 70 \pm 17 \%, 62 \pm 16 \%$ and $78 \pm$ $17 \%$ of baseline, respectively, after antigen injection. In addition, most of the larger portal veins $(70-110 \mu \mathrm{m})$ also showed stenosis (diameters less than $100 \%$ of the predicted baseline value). This finding was consistent with that of the venography, in which the portal veins with diameters less than $300 \mu \mathrm{m}$ showed vanishment. Neither sinusoids nor central veins, however, exhibited demonstrable constriction or dilatation.

\section{DISCUSSION}

The purpose of the present study was to determine the anaphylactic hepatic venoconstrictive site in antigen-injected anesthetized rats. We performed not only portal venography in order to visualize all of the hepatic vascular tree except for the hepatic artery and hepatic vein, but also a histological investigation for further detailed information. The results from the portal venography revealed that the portal veins with a diameter less than $230 \pm 40 \mu \mathrm{m}$ could not be visualized, suggesting distal stenosis or obstruction, and that obvious stenosis was observed in portal veins with a diameter of $180-420 \mu \mathrm{m}$. To clarify more detailed changes in the portal venules distal to the vanished branches, we performed a liver histological analysis, the results of which suggested that the most portal venules with diameters less than $110 \mu \mathrm{m}$ showed a decrease in vessel caliber, while consistent stenosis was present in the $20-70 \mu \mathrm{m}$ portal venules during anaphylactic portal hypertension. Taken together, these findings suggest that hepatic venoconstriction occurs over a wide range of portal veins with the diameter up to $420 \mu \mathrm{m}$, and markedly in the portal venules with diameters less than $70 \mu \mathrm{m}$ during anaphylactic hypotension in anesthetized rats.

According to Ohm's law (V=IR), as applied to hemodynamic systems, the antigen-induced increase in Ppv in this study is accounted for by an increase in the R, but not the Q, because the blood flow to the liver decreases in this rat anaphylaxis model (22). Based on Pouseuille's law, $R=8 \eta \mathrm{L} / \pi \mathrm{r}^{4}$, where $\eta=$ 

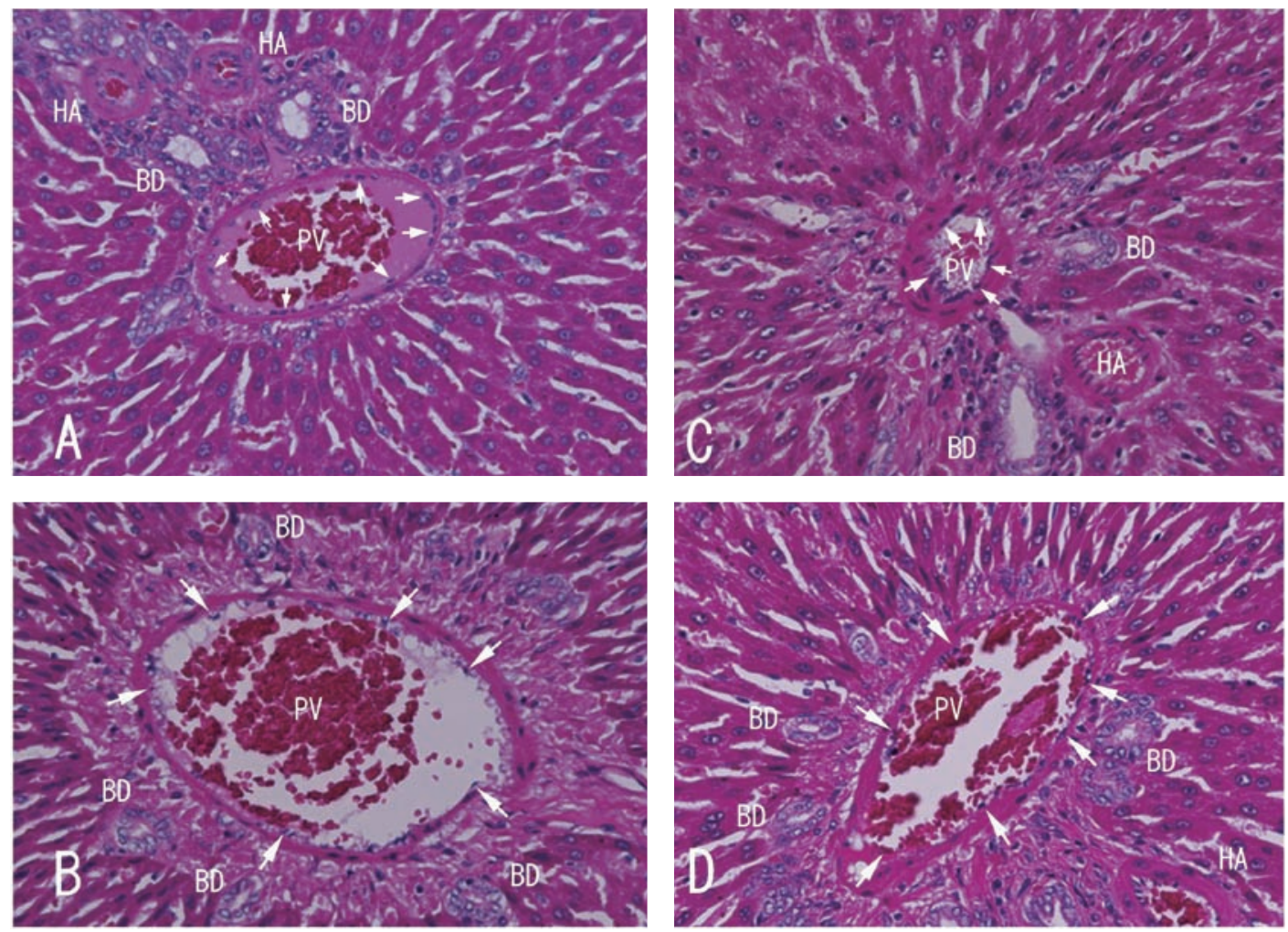

Fig. 3 Liver tissues obtained from non-sensitized rats (left panel in A, B) and sensitized (right panel in C, D) rats after an injection of the antigen $(\times 250)$. In $(A)$ and $(C)$, the portal veins with a 23 endothelial cell nuclei are shown, and in (B) and (D), those with 42 endothelial cell nuclei are shown. Arrows indicate endothelial cell nuclei. For the sake of simplicity, not all nuclei are shown. BD, bile duct; HA, hepatic artery; PV, portal vein.

viscosity coefficient, $\mathrm{L}=$ vessel length, and $\mathrm{r}=$ radius, $\mathrm{R}$ primarily depends on changes in $\mathrm{r}$, which doubtless will exert a dramatic influence over the definite resistance due to the fourth power. Thus, constriction of the small veins could cause a greater increase in resistance than that of the large veins, if the degree of constriction would be similar. Therefore, the markedly increased Ppv in the present study could be interpreted as arising from constriction of the portal venules with a diameter less than $70 \mu \mathrm{m}$, rather than constriction of the larger portal veins. With respect to the configuration of the intrahepatic portal system, which consists of conducting veins and distributing veins $(4,14)$, these $20-70 \mu \mathrm{m}$ portal venules may correspond to the distributing veins, including portal venules, which are the axial vessels of the complex acini and are localized at the corner of the liver lobule. On the other hand, the conducting veins consist of interlobar veins, segmental veins, and interlobular veins with a diameter of larger than $300 \mu \mathrm{m}$ (14). The present portal venography performed at maximal anaphylactic venoconstriction revealed that these conducting veins did not show stenosis but bulging. This finding was consistent with the functional feature of the conducting veins, which serve as conduit vessels with low vascular resistance.

Marked constriction has also been observed at a similar level in portal venules with a diameter of 40 to $80 \mu \mathrm{m}$ in in situ perfused rat livers administered a strong vasoconstrictor, endothelin-1; in these samples, the Ppv increased considerably, from 22 to 54 $\mathrm{cmH}_{2} \mathrm{O}$ (11). These data may suggest that the rat portal venous resistance site that is sensitive to vasoconstrictive substances resides at the distal portal venules. The distinct contraction of a certain vascular segment makes the mechanisms similar to the sphincter functionally. The portal veins that are responsible for the quasi-sphincter mechanism in rats seems to be portal venules with an inner diameter less than $70-80 \mu \mathrm{m}$. This portal venule may be analogous in a sense to the primary resistance vessel of the primary and secondary arterioles $(13-50 \mu \mathrm{m}$ internal diameter) in the systemic circulation $(7,12)$. However, further investigations for the portal venules with less than $70-80 \mu \mathrm{m}$ diameter as the por- 


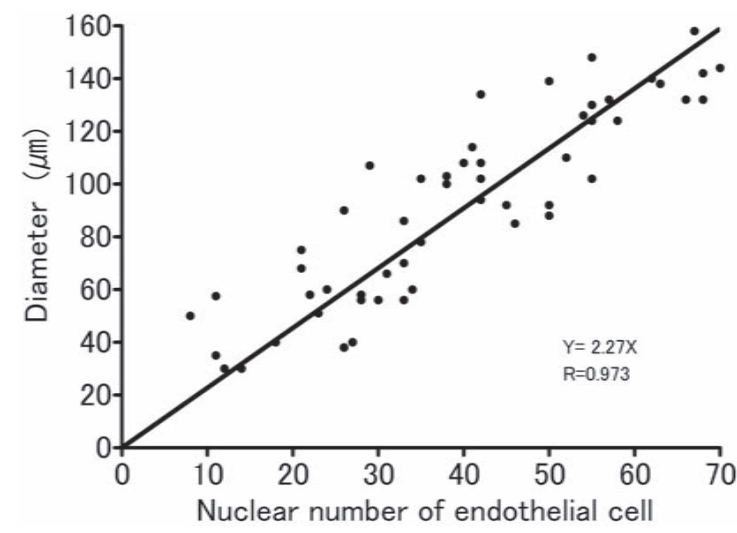

Fig. 4 The portal vein diameters measured as a function of the numbers of endothelial cell nuclei in the non-sensitized control rats. The regression line represents the equation $\mathrm{D}=2.27 \times \mathrm{N}$, where $\mathrm{D}$ and $\mathrm{N}$ indicate the diameter and nuclear number, respectively.

tal resistance site will need to be performed by verifying the consistent high responsiveness of this segment to the other vasoconstrictive substances. In addition, the rat hepatic constrictive site of the distal portal venules may not be applied to other species, such as dogs. Actually, in dogs, the post-sinusoidal hepatic veins contract at the sublobular sphincter in response to vasoconstrictors (21).

In the portal venography of the present study, the different vessel images of vanishment, stenosis, dilatation, and no change were observed in the 4th and 5 th generation branches. These findings may suggest the presence of heterogeneity of hepatic vasoreactivity. Some branches contracted strongly, resulting in stenosis, while some contracted modestly with resultant dilatation or no change. These differences depend on the balance between the increased distending pressure due to elevated Ppv and the strength of venoconstriction of the given portal vein branch. When the contractility overwhelmed the distending pressure, stenosis appeared, and when the former was overwhelmed by the latter, dilatation developed. The increased distending pressure of Ppv might be produced by strong constriction of the portal venules with a diameter less than $70 \mu \mathrm{m}$, because the vascular resistance of these segments might be extremely increased.

With respect to the mechanism for the hepatic venoconstriction during anaphylactic hypotension in the present study, there are two possibilities. The first is that the hepatic venoconstriction may have been caused by activation of the sympathetic nervous system. It is known that electrical stimulation of the rat hepatic sympathetic nerves evokes con-
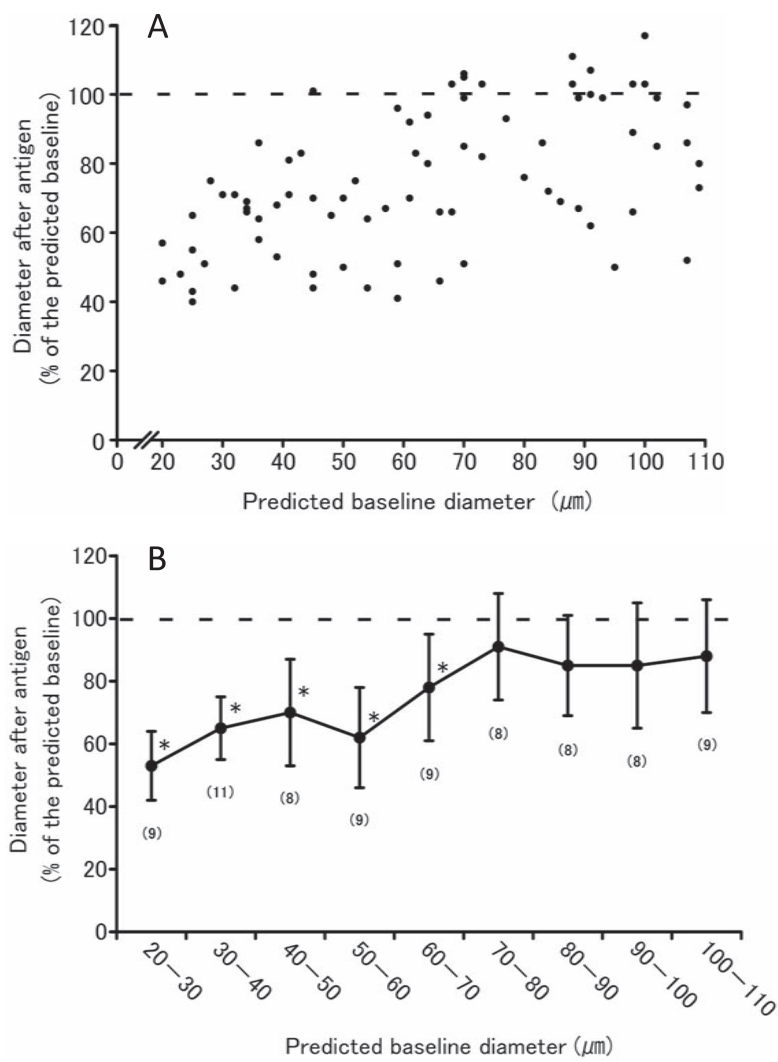

Fig. 5 (A) The plots of the portal vein diameters after antigen injection, which were expressed as a percentage of the predicted baseline diameters, as a function of the predicted baseline diameters in the anaphylaxis group. (B) The summarized portal vein diameters for the portal veins with predicted basal diameters from $20-30 \mu \mathrm{m}$ to $100-110 \mu \mathrm{m}$ in the anaphylaxis group. The results are shown as the means $\pm \mathrm{SD}$. $\mathrm{n}$ in the parenthesis, the numbers of the vessels examined. ${ }^{*} P<0.05$ vs. the basal predicted diameter $(100 \%)$.

striction of the hepatic vessels (1). On the other hand, renal sympathetic nerve activity was reported to increase in pentobarbital-anesthetized rats suffering from ovalbumin-induced anaphylactic hypotension (15). Furthermore, norepinephrine, the neurotransmitter released from the sympathetic nerve endings, can constrict presinusoidal vessel almost selectively, as demonstrated in the perfused rat livers with the vascular occlusion method (9). In addition, plasma levels of epinephrine and norepinephrine increased 14- and 4-fold baseline, respectively, at $2.5 \mathrm{~min}$ after antigen injection in a similar sensitized rat (23). Thus increased catecholamines might have constricted the hepatic vessels in the present study. Another possibility may be related to vasoactive chemical mediators released in response to the antigen. Cui et al. reported using isolated perfused rat livers that cysteinyl leukotrienes and cyclooxygenase products 
are mainly involved in anaphylactic venoconstriction (3). Furthermore, Shibamoto et al. showed using genetically mast cell-deficient rats that these anaphylactic vasoactive mediators may be released from the mast cells (25\%) and other cells (75\%) in anesthetized rats, and that mast cells may account for much of the hepatic anaphylactic reaction of the isolated perfused rat liver (17).

In conclusion, by using both venographic and morphological procedures in anesthetized rats suffering from systemic anaphylaxis, we demonstrated that the portal veins with diameters up to $420 \mu \mathrm{m}$ could contract, and that the portal venules with diameters of $20-70 \mu \mathrm{m}$ contracted markedly during hepatic anaphylactic venoconstriction.

\section{Acknowledgement}

This study was supported by Grants-in-Aid for Scientific Research (23592268, 24791342) from the Ministry of Education, Culture, Sports, Sciences and Technology of Japan, and Grants from Kanazawa Medical University (PR2012-13, C2011-2, SR201204).

\section{REFERENCES}

1. Beckh KH and Arnold R (1991) Regulation of bile secretion by sympathetic nerves in perfused rat liver. Am $J$ Physiol Gastrointest Liver Physiol 24, G775-G780.

2. Brown AFT (1995) Anaphylactic shock: mechanism and treatment. J Accid Emerg Med 12, 89-100.

3. Cui S, Shibamoto T, Takano H, Zhang W and Kurata Y (2007) Leukotrienes and cyclooxygenase products mediate anaphylactic venoconstriction in ovalbumin sensitized rat livers. Eur J Pharmacol 576, 99-106.

4. Elias H (1949) A re-examination of the structure of the mammalian liver. II. The hepatic lobule and its relation to the vascular and biliary systems. Am J Anat 85, 379-456.

5. Enjeti S, Bleecker ER, Smith PL, Rabson J, Permutt S and Traystman RJ (1983) Hemodynamic mechanism in anaphylactic shock. Circ Shock 11, 297-309.

6. Essex HE (1965) Anaphylactic and anaphylactoid reactions with special emphasis on the circulation. In Handbook of Physiology. Circulation III. Chapter 66. pp2391-2408, Am Physiol Soc, Bethesda, MD.

7. Fronek K and Zweifach BW (1975) Microvascular pressure distribution in skeletal muscle and the effect of vasodilation. Am J Physiol 228, 791-796.

8. Hines KL and Fisher R (1992) Regulation of hepatic glycogenolysis and vasoconstriction during antigen-induced anaphylaxis. Am J Physiol Gastrointest Liver Physiol 262, G868-G877.

9. Kamikado C, Shibamoto T, Hongo M and Koyama S (2004) Effects of hematocrit and norepinephrine on segmental vas- cular resistance distribution in isolated perfused rat livers. Am J Physiol Heart Circ Physiol 286, H121-H130.

10. Kamikado C, Shibamoto T, Zhang W, Kuda Y, Ohmukai C and Kurata Y (2011) Portacaval shunting attenuates portal hypertension and systemic hypotension in rat anaphylactic shock. J Physiol Sci 61, 161-166.

11. Kaneda K, Ekataksin W, Sogawa M, Matsumura A, Cho A and Kawada N (1998) Endothelin-1-induced vasoconstriction causes a significant increase in portal pressure of rat liver: localized constrictive effect on the distal segment of preterminal portal venules as revealed by light and electron microscopy and serial reconstruction. Hepatology 27, 735-747.

12. Marshall JM (1982) The influence of the sympathetic nervous system on individual vessels of the microcirculation of skeletal muscle of the rat. $J$ Physiol 332, 169-186.

13. Martins PNA and Neuhaus P (2007) Surgical anatomy of the liver, hepatic vasculature and bile ducts in the rat. Liver Int 27, 384-392.

14. Matsumoto T, Komori R, Magara T, Ui T, Kawakami T, Tokuda S, Takasaki S, Hayashi H, Jo K, Hano H, Fujino H and Tanaka H (1979) A study on the normal structure of the human liver, with special reference to its angioarchitecture. Jikeikai Med J 26, 1-40.

15. Potas JR, Briscoe H, Horiuchi J, Killinger S and Dampney RA (2004) Renal sympathetic and cardiac changes associated with anaphylactic hypotension. Auton Neurosci 112, 25-30.

16. Shibamoto T, Cui S, Ruan Z, Liu W, Takano H and Kurata Y (2005) Hepatic venoconstriction is involved in anaphylactic hypotension in rats. Am J Physiol Heart Circ Physiol 289, H1436-H1441.

17. Shibamoto T, Shimo T, Cui S, Zhang W, Takano H and Kurata Y (2007) The roles of mast cells and Kupffer cells in rat systemic anaphylaxis. Am J Physiol Regul Integr Comp Physiol 293, R2202-R2209.

18. Takano H, Shibamoto T, Zhang W, Kurata $Y$ and Toga $H$ (2009) Hepatic microvascular pressure during anaphylactic shock in anesthetized rats. Microvasc Res 78, 169-173.

19. Takano H, Shibamoto T, Zhang W and Kurata Y (2010) Liver volume, as assessed by four ultrasonic crystals arranged to form a tetrahedron, decreases during anaphylactic shock in anesthetized rats. Shock 34, 586-591.

20. Yamaguchi Y, Shibamoto T, Hayashi T, Saeki Y and Tanaka S (1994) Hepatic vascular response to anaphylaxis in isolated canine liver. Am J Physiol Regul Integr Comp Physiol 267, R268-R274.

21. Yamamoto K (1998) Ultrastructural study on the venous sphincter in the sublobular vein of the canine liver. Microvasc Res 55, 215-222.

22. Zhang W, Shibamoto T, Kurata Y, Shinomiya S, Kida M and Tsuchida H (2012) Vascular perfusion limits mesenteric lymph flow during anaphylactic hypotension in rats. Am J Physiol Regul Integr Comp Physiol 302, R1191-R1196.

23. Zhang W, Shibamoto T, Kuda Y, Ohmukai C and Kurata Y (2011) Pulmonary vasoconstrictive and bronchoconstrictive responses to anaphylaxis are weakened via $\beta_{2}$-adrenoceptor activation by endogenous epinephrine in anesthetized rats. Anesthesiology 114, 614-623.

24. Zhang W, Shibamoto T, Kuda Y, Shinomiya S and Kurata Y (2012) The responses of the hepatic and splanchnic vascular beds to vasopressin in rats. Biomed Res 33, 83-88. 